\title{
On Nondifferentiable Higher-Order Symmetric Duality in Multiobjective Programming Involving Cones
}

\author{
Xin Min Yang $\cdot$ Jin Yang $\cdot$ Tsz Leung Yip
}

Received: 31 October 2013 / Accepted: 25 November 2013 / Published online: 10 December 2013 (C) Operations Research Society of China, Periodicals Agency of Shanghai University, and Springer-Verlag Berlin Heidelberg 2013

\begin{abstract}
In this paper, we point out some deficiencies in a recent paper (Lee and Kim in J. Nonlinear Convex Anal. 13:599-614, 2012), and we establish strong duality and converse duality theorems for two types of nondifferentiable higher-order symmetric duals multiobjective programming involving cones.
\end{abstract}

Keywords Multiobjective programming - Higher-order Mond-Weir symmetric dual model · Higher-order Wolfe symmetric dual model · Strong duality theorems . Converse duality theorems · Nondifferentiability

\section{Introduction}

Mangasarian [4] first formulated a class of higher-order dual problems for nonlinear programming problems. Later, in [5], Mond and Weir gave the conditions for duality and considered other higher-order duals. Mond and Zhang [6] obtained duality results for various higher-order dual problems under higher-order invexity assumptions. Higher-order duality in nonlinear programming has been studied in the

This work was partially supported by the National Natural Science Foundation of China (Nos. 11271391 and 10831009) and the Natural Science Foundation of Chongqing (CSTC, No. 2011BA0030).

X.M. Yang $(\bowtie)$

Department of Mathematics, Chongqing Normal University, Chongqing 400047, China

e-mail: xmyang@cqnu.edu.cn

J. Yang

Department of Applied Mathematics, The Hong Kong Polytechnic University, Hung Hom, Kowloon, Hong Kong, China

T.L. Yip

Department of Logistics and Maritime Studies, The Hong Kong Polytechnic University, Hung Hom, Kowloon, Hong Kong, China 
last few years by many researchers. One practical advantage of higher-order duality is that it provides tighter bounds for the value of objective function of the primal problem when approximations are used because there are more parameters involved. Mishra and Rueda [8] considered higher-order duality for the nondifferentiable mathematical programming. They formulated a number of higher-order duals to a nondifferentiable programming problems and established duality under the higher-order generalized invexity conditions introduced in [7]. In [9], Yang et al. extended the results in [8] to a class of nondifferentiable multiobjective programs. Chen [2] studied higher-order symmetric duality for multiobjective nondifferentiable programs by introducing higher-order F-convexity. Agarwal et al. [1] extended the results of [2] to arbitrary cones and proved appropriate duality relations under higher-order K-Fconvexity assumptions.

Recently, Lee and Kim [3] have presented higher-order symmetric dual programs for multiobjective problems. In the literature strong and converse duality theorems have been established assuming conditions on known quantities. However, in strong and converse duality theorems in [3] an assumption involves the unknown Lagrange multiplier $\alpha$ for two types of symmetric duals. In this note we establish theses results under appropriate assumptions, this fills some gaps in the work of Lee and Kim [3].

\section{Notations and Preliminaries}

First we consider the following multiobjective programming problem:

$$
\begin{array}{ll}
\text { (P) } & \text { Minimize } \\
\text { subject to } & -g(x) \in Q, \quad x \in C,
\end{array}
$$

where $f: \mathbb{R}^{n} \rightarrow \mathbb{R}^{l}, g: \mathbb{R}^{n} \rightarrow \mathbb{R}^{m}, C \subset \mathbb{R}^{n}$ and $Q$ is a closed convex cone with nonempty interior in $\mathbb{R}^{m}$. We shall denote the feasible set of $(\mathbf{P})$ by

$$
X=\{x \mid-g(x) \in Q, x \in C\} .
$$

Definition 2.1 A nonempty set $K$ in $\mathbb{R}^{n}$ is said to be a cone with vertex zero, if $x \in K$ implies that $\lambda x \in K$ for all $\lambda \geqslant 0$. If, in addition, $K$ is convex, then $K$ is called a convex cone.

Definition 2.2 A feasible point $\bar{x}$ is a $K$-weakly efficient solution of (P), if there exists no other $x \in S$ such that $f(\bar{x})-f(x) \in$ int $K$.

Definition 2.3 The positive polar cone $K^{*}$ of $K$ is defined by

$$
K^{*}=\left\{z \in \mathbb{R}^{n} \mid x^{T} z \geqslant 0 \text { for all } x \in K\right\} .
$$

Definition 2.4 Let $S \subseteq \mathbb{R}^{n}$ be open and $F: S \rightarrow \mathbb{R}$ be a differentiable function. The function $F: S \rightarrow \mathbb{R}$ is said to be higher-order invex at $u \in S$ with respect to $\eta$ : $S \times S \rightarrow \mathbb{R}^{n}$ and $h: S \times \mathbb{R}^{n} \rightarrow \mathbb{R}$, if for all $(x, p) \in S \times \mathbb{R}^{n}$,

$$
f(x)-f(u) \geqslant \eta(x, u)^{T}\left[\nabla_{x} f(u)+\nabla_{p} h(u, p)\right]+h(u, p)-p^{T} \nabla_{p} h(u, p) .
$$


Definition 2.5 Let $C$ be a compact convex set in $\mathbb{R}^{n}$. The support function $s(x \mid C)$ of $C$ is defined by

$$
s(x \mid C):=\max \left\{x^{T} y: y \in C\right\} .
$$

The support function $s(x \mid C)$, being convex and everywhere finite, has a subdifferential, that is, there exists $z$ such that

$$
s(y \mid C) \geqslant s(x \mid C)+z^{T}(y-x), \quad \text { for all } x .
$$

The subdifferential of $s(x \mid C)$ is given by

$$
\partial s(x \mid C):=\left\{z \in C: z^{T} x=s(x \mid C)\right\} .
$$

For any set $S \subset \mathbb{R}^{n}$, the normal cone to $S$ at a point $x \in S$ is defined by

$$
N_{S}(x):=\left\{y \in \mathbb{R}^{n}: y^{T}(z-x) \leqslant 0 \text { for all } z \in S\right\} .
$$

It is readily verified that for a compact convex set $C, y$ is in $N_{S}(x)$ if and only if $s(y \mid C)=x^{T} y$, or equivalently, $x$ is in the subdifferential of $s$ at $y$.

Lemma 2.1 ([3]) If $\bar{x}$ is a $K$-weakly efficient solution of (P), then there exist $\alpha \in K^{*}$, $\beta \in Q^{*}$ not both zero such that

$$
\begin{gathered}
\left(\alpha^{T} \nabla f(\bar{x})+\beta^{T} \nabla g(\bar{x})\right)(x-\bar{x}) \geqslant 0, \quad \text { for all } x \in C, \\
\beta^{T} g(\bar{x})=0 .
\end{gathered}
$$

Equivalently, there exist $\alpha \in K^{*}, \beta \in Q^{*}, \beta_{1} \in C^{*}$, and $\left(\alpha, \beta, \beta_{1}\right) \neq 0$ such that

$$
\begin{aligned}
\alpha^{T} \nabla f(\bar{x})+\beta^{T} \nabla g(\bar{x})-\beta_{1} I & =0, \\
\beta^{T} g(\bar{x}) & =0, \\
\beta_{1}^{T} \bar{x} & =0 .
\end{aligned}
$$

\section{Mond-Weir Type Higher-Order Symmetric Duality}

We now establish duality theorems for the following pair of higher-order Mond-Weir type nondifferentiable multiobjective programming problems:

(MHNP)

$$
\text { Minimize } \quad \begin{aligned}
P_{M} & (x, y, \lambda, z, p) \\
= & \left(f_{1}(x, y)+s\left(x \mid C_{1}\right)-y^{T} z_{1}+h_{1}\left(x, y, p_{1}\right)\right. \\
& -p_{1}^{T}\left[\nabla_{p_{1}} h_{1}\left(x, y, p_{1}\right)\right], \cdots, f_{l}(x, y)+s\left(x \mid C_{l}\right) \\
& \left.-y^{T} z_{l}+h_{l}\left(x, y, p_{l}\right)-p_{l}^{T}\left[\nabla_{p_{l}} h_{l}\left(x, y, p_{l}\right)\right]\right)
\end{aligned}
$$




$$
\begin{array}{ll}
\text { subject to } & -\left(\sum_{i=1}^{l} \lambda_{i}\left[\nabla_{y} f_{i}(x, y)-z_{i}+\nabla_{p_{i}} h_{i}\left(x, y, p_{i}\right)\right]\right) \in Q_{2}^{*}, \\
& y^{T} \sum_{i=1}^{l} \lambda_{i}\left[\nabla_{y} f_{i}(x, y)-z_{i}+\nabla_{p_{i}} h_{i}\left(x, y, p_{i}\right)\right] \geqslant 0, \\
& x \in Q_{1}, \quad z_{i} \in D_{i}, \quad i=1,2, \cdots, l, \\
\lambda & \in \text { int } K^{*}, \quad \lambda^{T} e=1
\end{array}
$$

(MHND)

$$
\begin{aligned}
& \text { Maximize } \quad D_{M}(u, v, \lambda, \omega, r) \\
& =\left(f_{1}(u, v)-s\left(v \mid D_{1}\right)+u^{T} \omega_{1}+g_{1}\left(u, v, r_{1}\right)\right. \\
& -r_{1}^{T}\left[\nabla_{r_{1}} g_{1}\left(u, v, r_{1}\right)\right], \cdots, f_{l}(u, v)+s\left(v \mid D_{l}\right)-u^{T} w_{l} \\
& \left.+g_{l}\left(u, v, r_{l}\right)-r_{l}^{T}\left[\nabla_{r_{l}} g_{l}\left(u, v, r_{l}\right)\right]\right) \\
& \text { subject to } \sum_{i=1}^{l} \lambda_{i}\left[\nabla_{x} f_{i}(u, v)+\omega_{i}+\nabla_{r_{i}} g_{i}\left(u, v, r_{i}\right)\right] \in Q_{1}^{*} \text {, } \\
& u^{T} \sum_{i=1}^{l} \lambda_{i}\left[\nabla_{x} f_{i}(u, v)+\omega_{i}+\nabla_{r_{i}} f_{i}\left(u, v, r_{i}\right)\right] \leqslant 0, \\
& v \in Q_{2}, \quad \omega_{i} \in C_{i}, \quad i=1,2, \cdots, l, \\
& \lambda \in \text { int } K^{*}, \quad \lambda^{T} e=1
\end{aligned}
$$

where $C_{i}$ and $D_{i}$ are compact convex sets in $\mathbb{R}^{n}$ and $\mathbb{R}^{m}$, respectively; $Q_{1}$ and $Q_{2}$ are closed convex cones in $\mathbb{R}^{n}$ and $\mathbb{R}^{m}$, respectively; $e=(1,1, \cdots, 1)^{T}$ is a vector in $\mathbb{R}^{l} ;$ and for each $i=1,2, \cdots, l, f_{i}: \mathbb{R}^{n} \times \mathbb{R}^{m} \longrightarrow \mathbb{R}, h_{i}: \mathbb{R}^{n} \times \mathbb{R}^{m} \times \mathbb{R}^{m} \longrightarrow \mathbb{R}$ and $g_{i}: \mathbb{R}^{n} \times \mathbb{R}^{m} \times \mathbb{R}^{n} \longrightarrow \mathbb{R}$ are differentiable functions.

Any problem, say (MHND), in which $\lambda$ is fixed to be $\bar{\lambda}$, will be denoted by $(\mathrm{MHND})_{\bar{\lambda}}$.

Now we present a strong duality theorem for the dual pair (MHNP) and (MHND).

Theorem 3.1 (Strong Duality) Let $\left(\bar{x}, \bar{y}, \bar{\lambda}, \bar{z}_{1}, \bar{z}_{2}, \cdots, \bar{z}_{l}, \bar{p}_{1}, \bar{p}_{2}, \cdots, \bar{p}_{l}\right)$ be a $K$ weakly efficient solution of (MHNP). Assume that for each $i=1, \cdots, l, f_{i}: \mathbb{R}^{n} \times$ $\mathbb{R}^{m} \longrightarrow \mathbb{R}$ is thrice differentiable at $(\bar{x}, \bar{y}), h_{i}: \mathbb{R}^{n} \times \mathbb{R}^{m} \times \mathbb{R}^{m} \longrightarrow \mathbb{R}$ is twice differentiable at $\left(\bar{x}, \bar{y}, \bar{p}_{i}\right)$, and $g_{i}: \mathbb{R}^{n} \times \mathbb{R}^{m} \times \mathbb{R}^{n} \longrightarrow \mathbb{R}$ are differentiable functions. Furthermore, suppose that the following conditions are satisfied.

(I) For each $i=1,2, \cdots, l, h_{i}(\bar{x}, \bar{y}, 0)=0, g_{i}(\bar{x}, \bar{y}, 0)=0, \nabla_{p_{i}} h_{i}(\bar{x}, \bar{y}, 0)=0$, $\nabla_{y} h_{i}(\bar{x}, \bar{y}, 0)=0$ and $\nabla_{x} h_{i}(\bar{x}, \bar{y}, 0)=\nabla_{r_{i}} g(\bar{x}, \bar{y}, 0)$;

(II) For each $i=1,2, \cdots, l$, the Hessian matrix $\nabla_{p_{i} p_{i}} h_{i}\left(\bar{x}, \bar{y}, \bar{p}_{i}\right)$ is nonsingular; 
(III) The set of vectors $\left\{\nabla_{y} f_{i}(\bar{x}, \bar{y})-\bar{z}_{i}+\nabla_{p_{i}} h_{i}\left(\bar{x}, \bar{y}, \bar{p}_{i}\right)\right\}_{i=1}^{l}$ is linearly independent;

(IV) $\nabla_{y} h_{i}\left(\bar{x}, \bar{y}, \bar{p}_{i}\right)=\nabla_{p_{i}} h_{i}\left(\bar{x}, \bar{y}, \bar{p}_{i}\right), i=1,2, \cdots, l$;

(V) $K$ is a closed convex cone with $\mathbb{R}_{+}^{l} \subseteq K$.

Then, we have

(i) $\bar{p}_{i}=0, i=1,2, \cdots, l$;

(ii) there exists $\bar{\omega}_{i} \in C_{i}, i=1,2, \cdots, l$, such that $\left(\bar{x}, \bar{y}, \bar{\lambda}, \bar{\omega}_{1}, \bar{\omega}_{2}, \cdots, \bar{\omega}_{l}, \bar{r}_{1}=\bar{r}_{2}=\right.$ $\cdots=\bar{r}_{l}=0$ ) is a feasible solution of (MHND).

Furthermore, if the hypotheses of Theorem 2.1 in [3] are satisfied, then $(\bar{x}, \bar{y}, \bar{\lambda}$, $\left.\bar{\omega}_{1}, \bar{\omega}_{2}, \cdots, \bar{\omega}_{l}, \bar{r}_{1}=\bar{r}_{2}=\cdots=\bar{r}_{l}=0\right)$ is a $K$-weakly efficient solution to (MHND), and the two objective values are equal.

Proof Since $(\bar{x}, \bar{y}, \bar{\lambda}, \bar{z}, \bar{p})$ is a $K$-weakly efficient solution of (MHNP), by Lemma 2.1, then there exist $\alpha \in K^{*}, \beta \in Q_{2}, \mu \in \mathbb{R}_{+}, \delta \in Q_{1}^{*}$ and $\rho \in K$ such that

$$
\begin{aligned}
& \sum_{i=1}^{l} \alpha_{i}\left[\nabla_{x} f_{i}(\bar{x}, \bar{y})+w_{i}+\nabla_{x} h_{i}\left(\bar{x}, \bar{y}, \bar{p}_{i}\right)\right]+\sum_{i=1}^{l} \bar{\lambda}_{i}(\beta-\mu \bar{y})^{T} \nabla_{y x} f_{i}(\bar{x}, \bar{y}) \\
& +\sum_{i=1}^{l}\left(\bar{\lambda}_{i} \beta-\bar{\lambda}_{i} \mu \bar{y}-\alpha_{i} \bar{p}_{i}\right)^{T} \nabla_{p_{i} x} h_{i}\left(\bar{x}, \bar{y}, \bar{p}_{i}\right)-\delta=0, \\
& \sum_{i=1}^{l} \alpha_{i}\left[\nabla_{y} f_{i}(\bar{x}, \bar{y})-\bar{z}_{i}+\nabla_{y} h_{i}\left(\bar{x}, \bar{y}, \bar{p}_{i}\right)\right] \\
& -\mu \sum_{i=1}^{l} \bar{\lambda}_{i}\left[\nabla_{y} f_{i}(\bar{x}, \bar{y})-\bar{z}_{i}+\nabla_{p_{i}} h_{i}\left(\bar{x}, \bar{y}, \bar{p}_{i}\right)\right] \\
& +\sum_{i=1}^{l}\left(\bar{\lambda}_{i} \beta-\bar{\lambda}_{i} \mu \bar{y}-\alpha_{i} \bar{p}_{i}\right)^{T} \nabla_{p_{i} y} h_{i}\left(\bar{x}, \bar{y}, \bar{p}_{i}\right) \\
& +\sum_{i=1}^{l} \bar{\lambda}_{i}(\beta-\mu \bar{y})^{T} \nabla_{y y} f_{i}(\bar{x}, \bar{y})=0, \\
& (\beta-\mu \bar{y})^{T}\left[\nabla_{y} f_{i}(\bar{x}, \bar{y})-\bar{z}_{i}+\nabla_{p_{i}} h_{i}\left(\bar{x}, \bar{y}, \bar{p}_{i}\right)\right]-\rho_{i}=0, \quad i=1, \cdots, l, \\
& \left(\bar{\lambda}_{i} \beta-\bar{\lambda}_{i} \mu \bar{y}-\alpha_{i} \bar{p}_{i}\right)^{T} \nabla_{p_{i} p_{i}} h_{i}\left(\bar{x}, \bar{y}, \bar{p}_{i}\right)=0, \quad i=1, \cdots, l, \\
& \beta^{T} \sum_{i=1}^{l} \bar{\lambda}_{i}\left[\nabla_{y} f_{i}(\bar{x}, \bar{y})-\bar{z}_{i}+\nabla_{p_{i}} h_{i}\left(\bar{x}, \bar{y}, \bar{p}_{i}\right)\right]=0, \\
& \mu \bar{y}^{T} \sum_{i=1}^{l} \bar{\lambda}_{i}\left[\nabla_{y} f_{i}(\bar{x}, \bar{y})-\bar{z}_{i}+\nabla_{p_{i}} h_{i}\left(\bar{x}, \bar{y}, \bar{p}_{i}\right)\right]=0,
\end{aligned}
$$




$$
\begin{aligned}
& \bar{x}^{T} \delta=0, \\
& \bar{\lambda}^{T} \rho=0, \\
& \alpha_{i} \bar{y}+\bar{\lambda}_{i} \beta-\bar{\lambda}_{i} \mu \bar{y} \in N_{D_{i}}\left(\bar{z}_{i}\right), \quad i=1, \cdots, l, \\
& \bar{x}^{T} w_{i}=s\left(\bar{x} \mid C_{i}\right), \quad w_{i} \in C_{i}, \quad i=1, \cdots, l, \\
& (\alpha, \beta, \mu, \delta, \rho) \neq 0 .
\end{aligned}
$$

By the assumption (II), (3.10) gives

$$
\bar{\lambda}_{i} \beta-\bar{\lambda}_{i} \mu \bar{y}-\alpha_{i} \bar{p}_{i}=0, \quad i=1, \cdots, l .
$$

Now Let $\alpha_{i}=0$ for some $i \in\{1,2, \cdots, l\}$. Since $R_{+}^{k} \subseteq K$ implies $K^{*} \subseteq K_{+}^{k}$. Therefore $\bar{\lambda}>0$. Then relation (3.18) yields $\beta=\mu \bar{y}$. From (3.8), the assumptions (IV) and (III), we obtain $\alpha_{i}=\mu \overline{\lambda_{i}}, i=1,2, \cdots, l$. That is, $\mu=0, \beta=0$ and $\alpha_{i}=0, i=$ $1,2, \cdots, l$. From (3.7) and (3.9), we have $\delta=0$ and $\rho=0$, respectively. These contradict (3.17). Thus, $\alpha_{i} \neq 0$, for all $i$. Since $\alpha \in K^{*}$ and $K^{*} \sqsubseteq \mathbb{R}_{+}^{k}$, therefore,

$$
\alpha_{i}>0, \quad i=1,2, \cdots, l .
$$

From $\bar{\lambda}>0$, (3.14) implies that $\rho=0$. (3.9) and the assumption (III), we have $\beta=\mu \bar{y}$. From (3.18) and $\alpha>0$, we know that $\bar{p}_{i}=0, i=1,2, \cdots, l$.

By (3.18) and the assumption (I), (3.7) and (3.8) give

$$
\sum_{i=1}^{l} \alpha_{i}\left[\nabla_{x} f_{i}(\bar{x}, \bar{y})+w_{i}+\nabla_{x} h_{i}\left(\bar{x}, \bar{y}, \bar{p}_{i}\right)\right]-\delta=0,
$$

and

$$
\sum_{i=1}^{l}\left(\alpha_{i}-\mu \bar{\lambda}_{i}\right)\left[\nabla_{y} f_{i}(\bar{x}, \bar{y})-\bar{z}_{i}+\nabla_{y} h_{i}\left(\bar{x}, \bar{y}, \bar{p}_{i}\right)\right]=0 .
$$

From the assumption (III), (3.20) implies $\alpha_{i}=\mu \bar{\lambda}_{i}, i=1, \cdots, l$. Thus, $\mu>0$, (3.19) holds, and we have

$$
\sum_{i=1}^{l} \mu \bar{\lambda}_{i}\left[\nabla_{x} f_{i}(\bar{x}, \bar{y})+w_{i}+\nabla_{x} h_{i}\left(\bar{x}, \bar{y}, \bar{p}_{i}\right)\right]-\delta=0
$$

and from the assumption (I), it becomes

$$
\sum_{i=1}^{l} \mu \bar{\lambda}_{i}\left[\nabla_{x} f_{i}(\bar{x}, \bar{y})+w_{i}+\nabla_{r_{i}} g_{i}\left(\bar{x}, \bar{y}, \bar{p}_{i}\right)\right]=\delta \in Q_{1}^{*} .
$$

Also, since $\mu>0$, it follows that

$$
\sum_{i=1}^{l} \bar{\lambda}_{i}\left[\nabla_{x} f_{i}(\bar{x}, \bar{y})+w_{i}+\nabla_{r_{i}} g_{i}\left(\bar{x}, \bar{y}, \bar{p}_{i}\right)\right] \in Q_{1}^{*} .
$$


And from (3.18), $\bar{y}=\frac{\beta}{\mu} \in Q_{2}$. Multiplying (3.21) by $\bar{x}$ and using (3.13), it gives

$$
\bar{x}^{T} \sum_{i=1}^{l} \bar{\lambda}_{i}\left[\nabla_{x} f_{i}(\bar{x}, \bar{y})+w_{i}+\nabla_{r_{i}} g_{i}\left(\bar{x}, \bar{y}, \bar{p}_{i}\right)\right]=0 .
$$

Taking $\bar{w}_{i}=w_{i}, i=1, \cdots, l$, we find that $(\bar{x}, \bar{y}, \bar{\lambda}, \bar{w}, \bar{r}=0)$ is a feasible solution of (MHND). Moreover, from (3.15), we get $\bar{y} \in N_{D_{i}}\left(\bar{z}_{i}\right), i=1, \cdots, l$, so that $\bar{y}^{T} \bar{z}_{i}=$ $s\left(\bar{y} \mid D_{i}\right), i=1, \cdots, l$. Consequently, using (3.16),

$$
\begin{aligned}
& f_{i}(\bar{x}, \bar{y})+s\left(\bar{x} \mid C_{i}\right)-\bar{y}^{T} \bar{z}_{i}+h_{i}\left(\bar{x}, \bar{y}, \bar{p}_{i}\right)-\bar{p}_{i}^{T} \nabla_{p_{i}} h_{i}\left(\bar{x}, \bar{y}, \bar{p}_{i}\right) \\
& \quad=f_{i}(\bar{x}, \bar{y})-s\left(\bar{y} \mid D_{i}\right)+\bar{x}^{T} \bar{w}_{i} \\
& \quad=f_{i}(\bar{x}, \bar{y})-s\left(\bar{y} \mid D_{i}\right)+\bar{x}^{T} \bar{w}_{i}+g_{i}\left(\bar{x}, \bar{y}, \bar{r}_{i}\right)-\bar{r}_{i}^{T} \nabla_{r_{i}} g_{i}\left(\bar{x}, \bar{y}, \bar{r}_{i}\right), \quad i=1,, \cdots, l .
\end{aligned}
$$

Therefore, the objective values of (MHNP) and (MHND) are equal. Also, by Theorem 2.1 in [3], $(\bar{x}, \bar{y}, \bar{\lambda}, \bar{z}, \bar{p}=0)$ and $(\bar{x}, \bar{y}, \bar{\lambda}, \bar{w}, \bar{r}=0)$ are $K$-weakly efficient solutions of (MHNP) and (MHND), respectively.

We now state a converse duality theorem whose proof follows on the line of Theorem 3.1.

Theorem 3.2 (Converse Duality) Let $\left(\bar{u}, \bar{v}, \bar{\lambda}, \bar{\omega}_{1}, \bar{\omega}_{2}, \cdots, \bar{\omega}_{l}, \bar{r}_{1}, \bar{r}_{2}, \cdots, \bar{r}_{l}\right)$ be a $K$-weakly efficient solution of (MHND). Assume that for each $i=1, \cdots, l, f_{i}$ : $\mathbb{R}^{n} \times \mathbb{R}^{m} \longrightarrow \mathbb{R}$ is thrice differentiable at $(\bar{u}, \bar{v}), h_{i}: \mathbb{R}^{n} \times \mathbb{R}^{m} \times \mathbb{R}^{m} \longrightarrow \mathbb{R}$ is twice differentiable at $\left(\bar{u}, \bar{v}, \bar{r}_{i}\right)$, and $g_{i}: \mathbb{R}^{n} \times \mathbb{R}^{m} \times \mathbb{R}^{n} \longrightarrow \mathbb{R}$ are differentiable functions. Furthermore, suppose that the following conditions are satisfied.

(I) For each $i=1,2, \cdots, l, h_{i}(\bar{u}, \bar{v}, 0)=0, g_{i}(\bar{u}, \bar{v}, 0)=0, \nabla_{r_{i}} h_{i}(\bar{u}, \bar{v}, 0)=0$, $\nabla_{v} h_{i}(\bar{u}, \bar{v}, 0)=0$ and $\nabla_{u} h_{i}(\bar{u}, \bar{v}, 0)=\nabla_{r_{i}} g(\bar{u}, \bar{v}, 0)$

(II) For each $i=1,2, \cdots, l$, the Hessian matrix $\nabla_{r_{i} r_{i}} h_{i}\left(\bar{u}, \bar{v}, \bar{r}_{i}\right)$ is nonsingular;

(III) The set of vectors $\left\{\nabla_{v} f_{i}(\bar{u}, \bar{v})-\bar{\omega}_{i}+\nabla_{r_{i}} h_{i}\left(\bar{u}, \bar{v}, \bar{r}_{i}\right)\right\}_{i=1}^{l}$ is linearly independent;

(IV) $\nabla_{v} h_{i}\left(\bar{u}, \bar{v}, \bar{r}_{i}\right)=\nabla_{r_{i}} h_{i}\left(\bar{u}, \bar{v}, \bar{r}_{i}\right), i=1,2, \cdots, l$;

(V) $K$ is a closed convex cone with $\mathbb{R}_{+}^{l} \subseteq K$.

Then, we have

(i) $\bar{r}_{i}=0, i=1,2, \cdots, l$; and

(ii) there exists $\bar{z}_{i} \in C_{i}, i=1,2, \cdots, l$, such that $\left(\bar{u}, \bar{v}, \bar{\lambda}, \bar{z}_{1}, \bar{z}_{2}, \cdots, \bar{z}_{l}, \bar{p}_{1}=\bar{p}_{2}=\right.$ $\left.\cdots=\bar{p}_{l}=0\right)$ is a feasible solution of (MHNP).

Furthermore, if the hypotheses of Theorem 2.1 in [3] are satisfied, then $(\bar{x}, \bar{y}, \bar{\lambda}$, $\bar{z}_{1}, \bar{z}_{2}, \cdots, \bar{z}_{l}, \bar{p}_{1}=\bar{p}_{2}=\cdots=\bar{p}_{l}=0$ ) is a $K$-weakly efficient solution to (MHNP), and the two objective values are equal.

\section{Wolfe Type Higher-Order Symmetric Duality}

We now establish duality theorems for the following pair of higher-order Wolfe type nondifferentiable multiobjective programming problems: 
(WHNP)

Minimize $P_{W}(x, y, \nabla, z, p)$

$$
\begin{aligned}
= & \left(f_{1}(x, y)+s\left(x \mid C_{1}\right)-y^{T} z_{1}+h_{1}\left(x, y, p_{1}\right)\right. \\
& -p_{1}^{T}\left[\nabla_{p_{1}} h_{1}\left(x, y, p_{1}\right)\right] \\
& -y^{T} \sum_{i=1}^{l} \lambda_{i}\left[\nabla_{y} f_{i}(x, y)-z_{i}+\nabla_{p_{i}} h_{i}\left(x, y, p_{i}\right)\right], \cdots, \\
& f_{l}(x, y)+s\left(x \mid C_{l}\right)-y^{T} z_{k}+h_{l}\left(x, y, p_{l}\right)-p_{l}^{T}\left[\nabla_{p_{l}} h_{l}\left(x, y, p_{l}\right)\right] \\
& \left.-y^{T} \sum_{i=1}^{l} \lambda_{i}\left[\nabla_{y} f_{i}(x, y)-z_{i}+\nabla_{p_{i}} h_{i}\left(x, y, p_{i}\right)\right]\right)
\end{aligned}
$$

subject to $\quad-\left(\sum_{i=1}^{l} \lambda_{i}\left[\nabla_{y} f_{i}(x, y)-z_{i}+\nabla_{p_{i}} h_{i}\left(x, y, p_{i}\right)\right]\right) \in Q_{2}^{*}$,

$x \in Q_{1}, \quad z_{i} \in D_{i}, \quad i=1,2, \cdots, k, \quad \lambda \in K^{*}, \quad \lambda^{T} e=1$

\section{(WHND)}

Maximize $\quad D_{W}(u, v, \nabla, w, r)$

$$
\begin{aligned}
= & \left(f_{1}(u, v)-s\left(v \mid D_{1}\right)+u^{T} \omega_{1}+g_{1}\left(u, v, r_{1}\right)\right. \\
& -r_{1}^{T}\left[\nabla_{r_{1}} g_{1}\left(u, v, r_{1}\right)\right] \\
& -u^{T} \sum_{i=1}^{l} \lambda_{i}\left[\nabla_{x} f_{i}(u, v)+\omega_{i}+\nabla_{r_{i}} f_{i}\left(u, v, r_{i}\right)\right], \cdots, \\
& \left.f_{l}(u, v)-s\left(v \mid D_{l}\right)+u^{T} \omega_{l}+g_{l}\left(u, v, r_{l}\right)-r_{l}^{T}\left[\nabla_{r_{l}} g_{l}\left(u, v, r_{l}\right)\right]\right) \\
& -u^{T} \sum_{i=1}^{l} \lambda_{i}\left[\nabla_{x} f_{i}(u, v)+\omega_{i}+\nabla_{r_{i}} f_{i}\left(u, v, r_{i}\right)\right]
\end{aligned}
$$

subject to $\sum_{i=1}^{k} \lambda_{i}\left[\nabla_{x} f_{i}(u, v)+\omega_{i}+\nabla_{r_{i}} g_{i}\left(u, v, r_{i}\right)\right] \in Q_{1}^{*}$,

$v \in Q_{2}, \quad \omega_{i} \in C_{i}, \quad i=1,2, \cdots, k, \quad \lambda \in K^{*}, \quad \lambda^{T} e=1$ 
where $C_{i}$ and $D_{i}$ are compact convex sets in $\mathbb{R}^{n}$ and $\mathbb{R}^{m}$, respectively; $Q_{1}$ and $Q_{2}$ are closed convex cones in $\mathbb{R}^{n}$ and $\mathbb{R}^{m}$, respectively; $e=(1,1, \cdots, 1)^{T}$ is a vector in $\mathbb{R}^{l}$; and for each $i=1,2, \cdots, l, f_{i}: \mathbb{R}^{n} \times \mathbb{R}^{m} \longrightarrow \mathbb{R}, h_{i}: \mathbb{R}^{n} \times \mathbb{R}^{m} \times \mathbb{R}^{m} \longrightarrow \mathbb{R}$ and $g_{i}: \mathbb{R}^{n} \times \mathbb{R}^{m} \times \mathbb{R}^{n} \longrightarrow \mathbb{R}$ are differentiable functions.

Any problem, say (WHND), in which $\lambda$ is fixed to be $\bar{\lambda}$, will be denoted by $(\mathrm{WHND})_{\bar{\lambda}}$.

Theorem 4.1 (Strong Duality) Let $\left(\bar{x}, \bar{y}, \bar{\lambda}, \bar{z}_{1}, \bar{z}_{2}, \cdots, \bar{z}_{l}, \bar{p}_{1}, \bar{p}_{2}, \cdots, \bar{p}_{l}\right)$ be a $K$ weakly efficient solution of (WHNP). Fix $\lambda=\bar{\lambda}$ in (WHND). Assume that for each $i=1, \cdots, l, f_{i}: \mathbb{R}^{n} \times \mathbb{R}^{m} \longrightarrow \mathbb{R}$ is thrice differentiable at $(\bar{x}, \bar{y}), h_{i}: \mathbb{R}^{n} \times \mathbb{R}^{m} \times$ $\mathbb{R}^{m} \longrightarrow \mathbb{R}$ is twice differentiable at $\left(\bar{x}, \bar{y}, \bar{p}_{i}\right)$, and $g_{i}: \mathbb{R}^{n} \times \mathbb{R}^{m} \times \mathbb{R}^{n} \longrightarrow \mathbb{R}$ is a differentiable at $\left(\bar{x}, \bar{y}, \bar{p}_{i}\right)$. Furthermore, suppose that the following conditions are satisfied.

(I) $h_{i}(\bar{x}, \bar{y}, 0)=0, \quad g_{i}(\bar{x}, \bar{y}, 0)=0, \quad \nabla_{p_{i}} h_{i}(\bar{x}, \bar{y}, 0)=0, \quad \nabla_{y} h_{i}(\bar{x}, \bar{y}, 0)=0$, $\nabla_{x} h_{i}(\bar{x}, \bar{y}, 0)=\nabla_{r_{i}} g(\bar{x}, \bar{y}, 0), i=1,2, \cdots, l$;

(II) for all $i \in\{1,2, \cdots, l\}$, the Hessian matrix $\nabla_{p_{i} p_{i}} h_{i}\left(\bar{x}, \bar{y}, \bar{p}_{i}\right)$ is nonsingular;

(III) the set of vectors $\left\{\nabla_{y} f_{i}(\bar{x}, \bar{y})-\bar{z}_{i}+\nabla_{p_{i}} h_{i}\left(\bar{x}, \bar{y}, \bar{p}_{i}\right), i=1,2, \cdots, l\right\}$ is linearly independent;

(IV) $\nabla_{y} h_{i}\left(\bar{x}, \bar{y}, \bar{p}_{i}\right)=\nabla_{p_{i}} h_{i}\left(\bar{x}, \bar{y}, \bar{p}_{i}\right), i=1,2, \cdots, l$;

(V) $K$ is a closed convex cone with $\mathbb{R}_{+}^{k} \subseteq K$.

Then, we have

(i) $\bar{p}_{i}=0, i=1,2, \cdots, l$;

(ii) there exists $\bar{\omega}_{i} \in C_{i}, i=1,2, \cdots, k$, such that $\left(\bar{x}, \bar{y}, \bar{\lambda}, \bar{\omega}_{1}, \bar{\omega}_{2}, \cdots, \bar{\omega}_{l}, \bar{r}_{1}=\bar{r}_{2}=\right.$ $\cdots=\bar{r}_{l}=0$ ) is a feasible solution for (WHND) and the corresponding values of (WHNP) and (WHND) are equal.

Furthermore, if the hypotheses of Theorem 3.1 in [3] are satisfied, then $(\bar{x}, \bar{y}, \bar{\lambda}$, $\bar{\omega}_{1}, \bar{\omega}_{2}, \cdots, \bar{\omega}_{l}, \bar{r}_{1}=\bar{r}_{2}=\cdots=\bar{r}_{l}=0$ ) is a K-weakly efficient solution of (WHND).

Proof Since $(\bar{x}, \bar{y}, \bar{\lambda}, \bar{z}, \bar{p})$ is a $K$-weakly efficient solution of (WHNP), by Lemma 2.1, then there exist $\alpha \in K^{*}, \beta \in Q_{2}, \delta \in Q_{1}^{*}$, and $\rho \in K$ such that

$$
\begin{aligned}
& \sum_{i=1}^{l} \alpha_{i}\left[\nabla_{x} f_{i}(\bar{x}, \bar{y})+w_{i}+\nabla_{x} h_{i}\left(\bar{x}, \bar{y}, \bar{p}_{i}\right)\right]+\sum_{i=1}^{l} \bar{\lambda}_{i}\left(\beta-\left(\alpha^{T} e\right) \bar{y}\right)^{T} \nabla_{y x} f_{i}(\bar{x}, \bar{y}) \\
& \quad+\sum_{i=1}^{l}\left(\bar{\lambda}_{i} \beta-\bar{\lambda}_{i}\left(\alpha^{T} e\right) \bar{y}-\alpha_{i} \bar{p}_{i}\right)^{T} \nabla_{p_{i} x} h_{i}\left(\bar{x}, \bar{y}, \bar{p}_{i}\right)-\delta=0 \\
& \sum_{i=1}^{l} \alpha_{i}\left[\nabla_{y} f_{i}(\bar{x}, \bar{y})-\bar{z}_{i}+\nabla_{y} h_{i}\left(\bar{x}, \bar{y}, \bar{p}_{i}\right)\right] \\
& \quad-\sum_{i=1}^{l} \bar{\lambda}_{i}\left(\alpha^{T} e\right)\left[\nabla_{y} f_{i}(\bar{x}, \bar{y})-\bar{z}_{i}+\nabla_{p_{i}} h_{i}\left(\bar{x}, \bar{y}, \bar{p}_{i}\right)\right]
\end{aligned}
$$




$$
\begin{aligned}
& \quad+\sum_{i=1}^{l}\left(\bar{\lambda}_{i} \beta-\bar{\lambda}_{i}\left(\alpha^{T} e\right) \bar{y}-\alpha_{i} \bar{p}_{i}\right)^{T} \nabla_{p_{i} y} h_{i}\left(\bar{x}, \bar{y}, \bar{p}_{i}\right) \\
& \quad+\sum_{i=1}^{l} \bar{\lambda}_{i}\left(\beta-\left(\alpha^{T} e\right) \bar{y}\right)^{T} \nabla_{y y} f_{i}(\bar{x}, \bar{y})=0 \\
& \left(\beta-\left(\alpha^{T} e\right)_{\bar{y}}\right)^{T}\left[\nabla_{y} f_{i}(\bar{x}, \bar{y})-\bar{z}_{i}+\nabla_{p_{i}} h_{i}\left(\bar{x}, \bar{y}, \bar{p}_{i}\right)\right]-\rho_{i}=0, \quad i=1, \cdots, l \\
& \left(\bar{\lambda}_{i} \beta-\bar{\lambda}_{i}\left(\alpha^{T} e\right) \bar{y}-\alpha_{i} \bar{p}_{i}\right)^{T} \nabla_{p_{i} p_{i}} h_{i}\left(\bar{x}, \bar{y}, \bar{p}_{i}\right)=0, \quad i=1, \cdots, l \\
& \beta^{T} \sum_{i=1}^{l} \bar{\lambda}_{i}\left[\nabla_{y} f_{i}(\bar{x}, \bar{y})-\bar{z}_{i}+\nabla_{p_{i}} h_{i}\left(\bar{x}, \bar{y}, \bar{p}_{i}\right)\right]=0, \\
& \bar{x}^{T} \delta=0, \\
& \bar{\lambda}^{T} \rho=0, \\
& \alpha_{i} \bar{y}+\bar{\lambda}_{i} \beta-\bar{\lambda}_{i}\left(\alpha^{T} e\right) \bar{y} \in N_{D_{i}}\left(\bar{z}_{i}\right), \quad i=1, \cdots, l \\
& \bar{x}^{T} w_{i}=s\left(\bar{x} \mid C_{i}\right), \quad w_{i} \in C_{i}, \quad i=1, \cdots, l \\
& (\alpha, \beta, \delta, \rho) \neq 0 .
\end{aligned}
$$

By the assumption (II), (4.8) gives

$$
\bar{\lambda}_{i} \beta-\bar{\lambda}_{i}\left(\alpha^{T} e\right) \bar{y}-\alpha_{i} \bar{p}_{i}=0, \quad i=1, \cdots, l .
$$

We claim that $\alpha=\left(\alpha_{1}, \cdots, \alpha_{l}\right)^{T} \neq 0$. Suppose that $\alpha=0$. Then, (4.15) yields $\beta=0$. From (4.5) and (4.7), we have $\delta=0$ and $\rho=0$. These contradict (4.14). Hence $\alpha \neq 0$.

If some $\alpha_{i}=0$, from $\bar{\lambda}_{i}>0, i=1,2, \cdots, l$, we know that (4.15) implies

$$
\beta=\left(\alpha^{T} e\right) \bar{y} .
$$

By the assumption (IV) and (4.16), (4.6) gives

$$
\sum_{i=1}^{l}\left(\alpha_{i}-\bar{\lambda}_{i}\left(\alpha^{T} e\right)\right)\left[\nabla_{y} f_{i}(\bar{x}, \bar{y})-\bar{z}_{i}+\nabla_{y} h_{i}\left(\bar{x}, \bar{y}, \bar{p}_{i}\right)\right]=0 .
$$

From the assumption (III), (4.17) implies that

$$
\alpha_{i}=\bar{\lambda}_{i}\left(\alpha^{T} e\right), \quad i=1, \cdots, l .
$$

So, $\alpha_{i}=0, i=1,2, \cdots, l$. This is a contradiction with $\alpha \neq 0$. Thus, $\alpha>0$.

From $\bar{\lambda}>0$, (4.11) implies $\rho=0$. From (4.7) and the assumption (III), we have $\beta=\left(\alpha^{T} e\right) \bar{y}$, from (4.15), $\bar{\lambda}>0$ and $\alpha>0$ again, we obtain $\bar{p}_{i}=0, i=1,2, \cdots, l$. 
By (4.15) and the assumption (I), (4.5) and (4.6) give

$$
\sum_{i=1}^{l} \alpha_{i}\left[\nabla_{x} f_{i}(\bar{x}, \bar{y})+w_{i}+\nabla_{r_{i}} g_{i}\left(\bar{x}, \bar{y}, \bar{p}_{i}\right)\right]-\delta=0,
$$

and

$$
\sum_{i=1}^{l}\left(\alpha_{i}-\bar{\lambda}_{i}\left(\alpha^{T} e\right)\right)\left[\nabla_{y} f_{i}(\bar{x}, \bar{y})-\bar{z}_{i}+\nabla_{y} h_{i}\left(\bar{x}, \bar{y}, \bar{p}_{i}\right)\right]=0
$$

From the assumption (III), (4.19) implies that

$$
\alpha_{i}=\bar{\lambda}_{i}\left(\alpha^{T} e\right), \quad i=1, \cdots, l
$$

Thus, (4.18) holds

$$
\sum_{i=1}^{l} \bar{\lambda}_{i}\left(\alpha^{T} e\right)\left[\nabla_{x} f_{i}(\bar{x}, \bar{y})+w_{i}+\nabla_{r_{i}} g_{i}\left(\bar{x}, \bar{y}, \bar{p}_{i}\right)\right]=\delta \in Q_{1}^{*} .
$$

And, since $\alpha^{T} e>0$, it follows that

$$
\sum_{i=1}^{l} \bar{\lambda}_{i}\left[\nabla_{x} f_{i}(\bar{x}, \bar{y})+w_{i}+\nabla_{r_{i}} g_{i}\left(\bar{x}, \bar{y}, \bar{p}_{i}\right)\right] \in Q_{1}^{*} .
$$

Also, from (4.15), $\bar{y}=\frac{\beta}{\alpha^{T} e} \in Q_{2}$. Taking $\bar{w}_{i}=w_{i}, i=1, \cdots, l$, we find that $(\bar{x}, \bar{y}, \bar{\lambda}, \bar{w}, \bar{r}=0)$ is a feasible solution of (WHND). Moreover, from (4.12), we get $\bar{y} \in N_{D_{i}}\left(\bar{z}_{i}\right), i=1, \cdots, l$, so that $\bar{y}^{T} \bar{z}_{i}=s\left(\bar{y} \mid D_{i}\right), i=1, \cdots, l$. Multiplying (4.20) by $\bar{x}$ and using (4.10), we have

$$
\bar{x}^{T} \sum_{i=1}^{l} \bar{\lambda}_{i}\left[\nabla_{x} f_{i}(\bar{x}, \bar{y})+w_{i}+\nabla_{r_{i}} g_{i}\left(\bar{x}, \bar{y}, \bar{p}_{i}\right)\right]=0 .
$$

And from (4.9) and (4.15), we obtain

$$
\bar{y}^{T} \sum_{i=1}^{l} \bar{\lambda}_{i}\left[\nabla_{y} f_{i}(\bar{x}, \bar{y})-z_{i}+\nabla_{p_{i}} h_{i}\left(\bar{x}, \bar{y}, \bar{p}_{i}\right)\right]=0 .
$$

Consequently, using (4.13),

$$
\begin{aligned}
& f_{i}(\bar{x}, \bar{y})+s\left(\bar{x} \mid C_{i}\right)-\bar{y}^{T} \bar{z}_{i}+h_{i}\left(\bar{x}, \bar{y}, \bar{p}_{i}\right)-\bar{p}_{i}^{T} \nabla_{p_{i}} h_{i}\left(\bar{x}, \bar{y}, \bar{p}_{i}\right) \\
& \quad-\bar{y}^{T} \sum_{i=1}^{l} \bar{\lambda}_{i}\left[\nabla_{y} f_{i}(\bar{x}, \bar{y})-z_{i}+\nabla_{p_{i}} h_{i}\left(\bar{x}, \bar{y}, \bar{p}_{i}\right)\right] \\
& =f_{i}(\bar{x}, \bar{y})-s\left(\bar{y} \mid D_{i}\right)+\bar{x}^{T} \bar{w}_{i}
\end{aligned}
$$




$$
\begin{aligned}
= & f_{i}(\bar{x}, \bar{y})-s\left(\bar{y} \mid D_{i}\right)+\bar{x}^{T} \bar{w}_{i}+g_{i}\left(\bar{x}, \bar{y}, \bar{r}_{i}\right)-\bar{r}_{i}^{T} \nabla_{r_{i}} g_{i}\left(\bar{x}, \bar{y}, \bar{r}_{i}\right) \\
& -\bar{x}^{T} \sum_{i=1}^{l} \bar{\lambda}_{i}\left[\nabla_{x} f_{i}(\bar{x}, \bar{y})+w_{i}+\nabla_{r_{i}} g_{i}\left(\bar{x}, \bar{y}, \bar{r}_{i}\right)\right], \quad i=1, \cdots, l .
\end{aligned}
$$

Therefore, the objective values of (WHNP) and (WHND) are equal. Also, by Theorem 3.1 in [3] $(\bar{x}, \bar{y}, \bar{\lambda}, \bar{z}, \bar{p}=0)$ and $(\bar{x}, \bar{y}, \bar{\lambda}, \bar{w}, \bar{r}=0)$ are $K$-weakly efficient solutions of (WHNP) and (WHND), respectively.

We now state a converse duality theorem whose proof follows on the line of Theorem 4.1.

Theorem 4.2 (Converse Duality) Let $\left(\bar{u}, \bar{v}, \bar{\lambda}, \bar{\omega}_{1}, \bar{\omega}_{2}, \cdots, \bar{\omega}_{l}, \bar{r}_{1}, \bar{r}_{2}, \cdots, \bar{r}_{l}\right)$ be a $K$-weakly efficient solution of (WHND). Assume that for each $i=1, \cdots, l, f_{i}$ : $\mathbb{R}^{n} \times \mathbb{R}^{m} \longrightarrow \mathbb{R}$ is thrice differentiable at $(\bar{u}, \bar{v}), h_{i}: \mathbb{R}^{n} \times \mathbb{R}^{m} \times \mathbb{R}^{m} \longrightarrow \mathbb{R}$ is twice differentiable at $\left(\bar{u}, \bar{v}, \bar{r}_{i}\right)$, and $g_{i}: \mathbb{R}^{n} \times \mathbb{R}^{m} \times \mathbb{R}^{n} \longrightarrow \mathbb{R}$ are differentiable functions. Furthermore, suppose that the following conditions are satisfied.

(I) For each $i=1,2, \cdots, l, h_{i}(\bar{u}, \bar{v}, 0)=0, g_{i}(\bar{u}, \bar{v}, 0)=0, \nabla_{r_{i}} h_{i}(\bar{u}, \bar{v}, 0)=0$, $\nabla_{v} h_{i}(\bar{u}, \bar{v}, 0)=0$ and $\nabla_{u} h_{i}(\bar{u}, \bar{v}, 0)=\nabla_{r_{i}} g(\bar{u}, \bar{v}, 0)$;

(II) For each $i=1,2, \cdots, l$, the Hessian matrix $\nabla_{r_{i} r_{i}} h_{i}\left(\bar{u}, \bar{v}, \bar{r}_{i}\right)$ is nonsingular;

(III) The set of vectors $\left\{\nabla_{v} f_{i}(\bar{u}, \bar{v})-\bar{\omega}_{i}+\nabla_{r_{i}} h_{i}\left(\bar{u}, \bar{v}, \bar{r}_{i}\right)\right\}_{i=1}^{l}$ is linearly independent;

(IV) $\nabla_{v} h_{i}\left(\bar{u}, \bar{v}, \bar{r}_{i}\right)=\nabla_{r_{i}} h_{i}\left(\bar{u}, \bar{v}, \bar{r}_{i}\right), i=1,2, \cdots, l$;

(V) $K$ is a closed convex cone with $\mathbb{R}_{+}^{l} \subseteq K$.

Then, we have

(i) $\bar{r}_{i}=0, i=1,2, \cdots, l$;

(ii) there exists $\bar{z}_{i} \in C_{i}, i=1,2, \cdots, l$, such that $\left(\bar{u}, \bar{v}, \bar{\lambda}, \bar{z}_{1}, \bar{z}_{2}, \cdots, \bar{z}_{l}, \bar{p}_{1}=\bar{p}_{2}=\right.$ $\left.\cdots=\bar{p}_{l}=0\right)$ is a feasible solution of (WHNP).

Furthermore, if the hypotheses of Theorem 3.1 in [3] are satisfied, then $(\bar{x}, \bar{y}, \bar{\lambda}$, $\bar{z}_{1}, \bar{z}_{2}, \cdots, \bar{z}_{l}, \bar{p}_{1}=\bar{p}_{2}=\cdots=\bar{p}_{l}=0$ ) is a K-weakly efficient solution to (WHNP), and the two objective values are equal.

\section{References}

[1] Agarwal, R.P., Ahmad, I., Gupta, S.K.: A note on higher order nondifferentiable symmetric duality in multiobjective programming. Appl. Math. Lett. 24, 308-1311 (2011)

[2] Chen, X.: Higher order symmetric duality in non-differentiable multiobjective programming problems. J. Math. Anal. Appl. 290, 423-435 (2004)

[3] Lee, Y.J., Kim, D.S.: Nondifferentiable higher order symmetric duality in multiobjective programming involving cones. J. Nonlinear Convex Anal. 13, 599-614 (2012)

[4] Mangasarian, O.L.: Second and higher order duality in nonlinear programming. J. Math. Anal. Appl. 51, 607-620 (1975)

[5] Mond, B., Weir, T.: Generalized convexity and higher order duality. J. Math. Sci. 16-18, 74-94 (19811983)

[6] Mond, B., Zhang, J.: Higher order invexity and duality in mathematical programming. In: Crouzeix, J.P., et al. (eds.) Generalized Convexity, Generalized Monotonicity: Recent Results, pp. 357-372. Kluwer Academic, Dordrecht/Norwell (1998) 
[7] Mishra, S.K., Rueda, N.G.: Higher-order generalized invexity and duality in mathematical programming. J. Math. Anal. Appl. 247, 173-182 (2000)

[8] Mishra, S.K., Rueda, N.G.: Higher-order generalized invexity and duality in nondifferentiable mathematical programming. J. Math. Anal. Appl. 272, 496-506 (2002)

[9] Yang, X.M., Teo, K.L., Yang, X.Q.: Higher order generalized convexity and duality in nondifferentiable multiobjective mathematical programming. J. Math. Anal. Appl. 297, 48-55 (2004) 